\title{
Identity Verification by Using Handprint
}

\author{
Hao Ying, Tan Tieniu, Sun Zhenan, and Han Yufei \\ National Laboratory of Pattern Recognition, \\ Institute of Automation, Chinese Academy of Sciences, China \\ \{yhao, tnt, znsun, yfhan\} @nlpr.ia.ac.cn
}

\begin{abstract}
In recent years, palmprint based personal identification has been extensively explored by researchers. The success of this technology has demonstrated that the inner part of palm skin is capable of distinguishing one person from another in case that proper representation is utilized. However, earlier work mainly focused on scenarios where the position and pose of hands are constrained by pegs or plates. In contrast, our purpose is to design and implement a system which is capable of recognizing an individual once he/she naturally stretches his/her hand in front of the camera. Since human hand is an articulated object, it is important to filter out geometry variations. This paper presents and compares two hand texture based personal identification methods, which are called hand-print verification in this paper to denote the idea of utilizing whole hand skin image for recognition. In one of the method, hand articulation is eliminated in a well-defined way and then the hand is treated as a whole for feature extraction, while in the other method, features are extracted in different parts of the hand, and final decision is made in a matching score level fusion manner. Experimental results for both methods are presented and compared.
\end{abstract}

Keywords: Biometrics, Verification, Identification, Ordinal Representation, Texture Analysis, Handprint.

\section{Introduction}

In our increasingly digitalized and connected society, personal impact expands from several meters to millions of miles. We all have a high probability of making conversation, communicating, sending email or even making business with people who connect with us from remote places via cable. In such an environment, identity is becoming more and more ambiguous. Determined criminals might threaten our life in dozens of ways, ranging from minor financial loss to personal safety. Biometrics has emerged as a novel way to establish identity by analyzing physical or behaviorial traits which have been recognized to be intrinsic to each person. Although palmprint recognition is a relatively young member of the Biometric family, its growth is considerably fast. Different approaches have been proposed. In our opinion, there are three categories of features that have been explored in the literature: 
- structural features, referring to line features, such as principal lines, wrinkles and even ridges,

- appearance based features, exploiting global texture by using linear subspace methods,

- texture features, adopting local textual information.

However, the above mentioned feature extraction schemes are all proposed for the scenarios where the position and/or pose of hands are constrained with pegs or plates, for example, scanner and dedicated device are most commonly adopted for palm image acquisition. What we want to do is to set free of the users from theses constraints by designing and implementing a system which is capable of recognizing an individual once he/she naturally stretches his/her hand in front of the camera. This is a much more difficult task compared with those in previous literature. For example, at least the following issues have to be considered:

- Localization. How to detect the presence of human hands? How to decide the precise orientation, location and scale of hands?

- Hand pose evaluation. How to evaluate hand images so that only hands with proper pose are selected for feature extraction?

- Preprocessing. How to process hand images so that ROIs (Region of Interest) are exactly located and hand articulation is eliminated?

- Feature extraction. How to make use of or modify previously successful feature extraction schemes?

Among the three categories of features, texture based approach is reported to be most powerful in distinguishing people. Representative work of this category [2], 5], 3] and [6], has been reporting better and better performance. These approaches share a common characteristics: a simple feature is extracted at each local region and a mesh containing one feature at each grid is utilized to provide a much more distinctive representation. Despite the success of this representation scheme, there is a major disadvantage: it does not contain a built-in mechanism to deal with partial skin deformation, such as the effect caused by stretching and scrunching, in which case the inner part of the palm changed considerably. In this paper, we seek to eliminate the variation of hand poses in preprocessing stage.

The texture of inner hand skin is affected by complex mechanism involved with hand bones, muscles and skin status, which make it difficult to model. However, if the anatomically meaningful points can be extracted from hand image, it is possible to compensate for the above mentioned non-linear deformation at preprocessing stage.

Besides palmprint, researchers have shown that finger surface regions also convey distinctive information [7], which motivates us to extend the concept of palmprint to a larger one: handprint, which denotes the idea of utilizing the whole hand skin image for recognition. Similar idea has been proposed in [8], where the authors present a method which utilizes grayscale distribution variance as features to describe non-normalized hands, which is inevitably sensitive to hand pose variations and illumination changes. 


\section{Proposed Methods}

We proposed two strategies to achieve the goal, one is a holistic method and the other is a fusion based method. The holistic method treats the hand as a whole and it is composed of the following steps:

1. A set of landmarks is defined and extracted by contour geometry as well as skin texture to provide geometrical information of hands;

2. The geometrical variation is filtered out by image warping;

3. Binary textual features are extracted to represent handprint;

4. Hamming distance is adopted to measure the similarity of two features.

While in fusion based method, feature extraction and matching are performed in a part by part manner, where a hand is partitioned to six parts, namely, palm region and five fingers. The matching results on different parts are finally combined at matching score level to make the final verification decision.

\subsection{Hand Landmark Localization}

Hand shapes, and hand contours accordingly, may change whenever hands move, rotate or hand pose varies. In order to analyze the hand texture as a whole, the shape variation must be somehow filtered out. One solution to this problem is to find out a representation of the hand shape and then transform this representation to a translation-, rotation- and pose-free one. A sparse landmark based approach can obviously meet this goal.

For hand images captured indoors under constrained environment, high quality hand contour can be easily obtained by any adaptive global thresholding algorithm followed by a boundary tracking method. By summing up all pixel values along the four thresholded image boundaries, the wrist position can be immediately recognized as the side that produces maximal sum value. After that, the middle of wrist is selected as reference point and distance from each contour point to the reference point is calculated, thus producing a one-dimensional profile. The five local maximums and four local minimums of this profile obviously correspond to finger tips and finger roots respectively. Then the starting point of thumb and index as well as end point of little finger are detected and fingers are separated by adopting the preprocessing method proposed by Xiong et. al. 4].

After the above process, 12 landmarks are detected. Although these landmarks are capable of characterizing hand shape coarsely, they do not meet the requirement of capturing hand shape variation.

Observation on hand images demonstrates that the pixel values near knuckle region are normally lower than other parts of fingers. This property makes it possible to extract knuckles and use them as landmarks. Fig. 11 shows the process of knuckle detection. Firstly, each separated finger image is rotated so that its principal axis becomes 90 degree. After that, histogram equalization is adopted to normalize contrast and enhance details. The mean pixel value along each horizontal line is then calculated and a one-dimensional signal is produced. The two local minimal points correspond to two knuckles of that finger, as illustrated 
in Fig. 1(c). The thumb knuckle normally locate near the middle of the whole finger while knuckles of other fingers usually lie close to $\frac{1}{3}$ and $\frac{2}{3}$ percent of the fingers. The above prior knowledge can be easily implemented in the localization process.

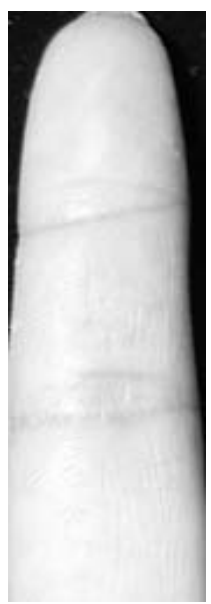

(a)

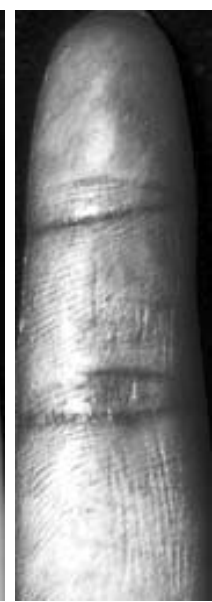

(b)

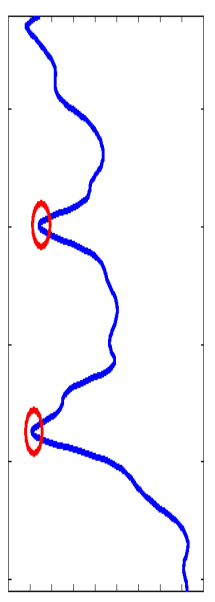

(c)

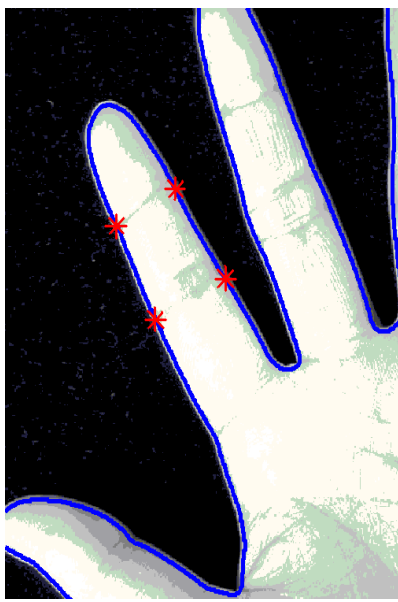

(d)

Fig. 1. Knuckle detection: (a) original finger image; (b)finger image after histogram equalization; (c) horizontal profile of (b) with the circles indicating the local minimums, and $(\mathrm{d})$ detected knuckle landmarks( stars) labeled on original hand image

The wrist beginning and ending point play important roles in defining the region of hand. However, they are much more subtle than other landmarks and more difficult to extract automatically, so we manually label them out.

The mean difference between proposed landmarks used here and those of Yörük et. al. 1 lies in that all the landmarks that we used here have straightforward anatomical meaning. This enables us to map one representation to another while minimizing the risk of introducing geometrical artifacts to hand shape.

\subsection{Holistic Method}

Image warping is an image processing method which moves image pixels from one spatial configurations to another. Digital image warping has benefited dramatically from several fields, ranging from early work in remote sensing to recent developments in computer graphics [9]. Basically, image warping involves two operations, namely mapping and resampling, where mapping defines destination for each source pixel and resampling decides the contribution of neighboring pixel values to the current one. Since the contour of each hand is defined by a set of landmarks, $\left\{\mathbf{x}_{1}, \mathbf{x}_{2}, \ldots, \mathbf{x}_{n}\right\}$, the simplest way to perform warping is to assume that the mapping function is locally linear. 
The Delaunay triangulation is a collection of lines that connect each point to its nearest neighbors, where the 'empty circle' property is satisfied: no points are contained in any triangle's circumscribed circle. The Delaunay triangulation of the mean hand contour is given in Fig. 2, where the convex hull of the hand is partitioned to a set of non-overlapping triangles.

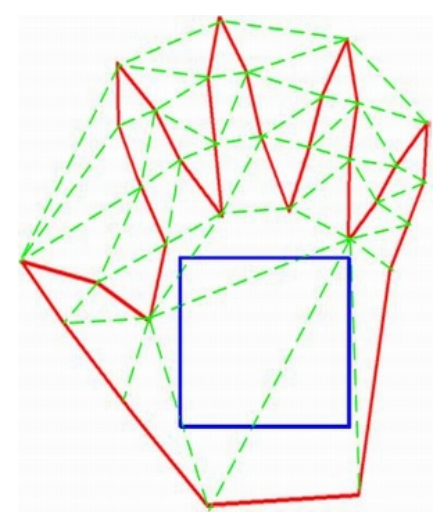

Fig. 2. Delaunay triangulation (dash lines) of mean hand landmarks

The warping is therefore implemented by transforming the triangles from one point set $\mathbf{P}$ to another point set $\mathbf{P}^{\prime}$. Since each triangle pair provides 3 pairs of correspondence, an affine transformation consisting of scaling, translation, rotation and shearing, can be uniquely decided. Suppose that $\mathbf{x}_{1}, \mathbf{x}_{2}, \mathbf{x}_{3}$ denote the three vertices of a certain triangle $T R$, and $\mathbf{x}_{1}^{\prime}, \mathbf{x}_{2}^{\prime}, \mathbf{x}_{3}^{\prime}$ denote vertices of the target triangle $T R^{\prime}$. Suppose that $\mathbf{x}$ lies in triangle $T R$, it can be represented as:

$$
\mathbf{x}=\alpha \mathbf{x}_{1}+\beta \mathbf{x}_{2}+(1-\alpha-\beta) \mathbf{x}_{3}
$$

then its target point satisfies:

$$
\mathbf{x}^{\prime}=\alpha \mathbf{x}_{1}^{\prime}+\beta \mathbf{x}_{2}^{\prime}+(1-\alpha-\beta) \mathbf{x}_{3}^{\prime}
$$

Therefore, each point in $T R$ can be mapped to the target triangle $T R^{\prime}$, which indicates that each hand can be mapped to the target one. In case that in-depth rotation of hand occurs or parts of hand are deformed by finger movement, this process helps to decrease intra-class differences.

\subsection{Fusion Based Method}

To deal with articulation of complex objects, a more flexible approach is to detect its constituent yet distinctive parts and combine cues from these parts to recognize objects. Motivated by this approach, we partition the hand into six meaningful parts: palm region and five finger regions. A rectangle region that 
covers approximately $80 \%$ percent of each finger is selected and resized to the average size of that finger. Palm region is denoted in Fig. 2 by the square of bold line.

Fig. 3 demonstrated the results of the above two methods, where the first row denotes four original hand images, the second row shows corresponding warping result and the last row indicates the segmented parts of each hand. Notice that anatomically meaningful regions are mapped to the same target region no matter what pose, location and angle it has on original images, which is obviously preferable in case of deformation. Since inter-finger regions are also processed in warping-based method, artifacts are introduced, especially when the inter-finger distances are small on original images, see Fig. 3(b)(f) and (d)(h) for examples. However, these artifacts do not propagate to feature-level representation, because we only extract feature within hand skin regions as indicated in Fig. 3(e)-(h).

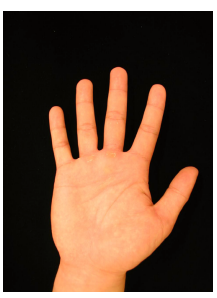

(a)

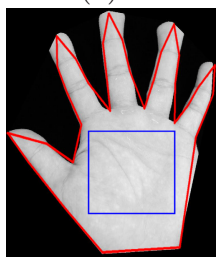

(e)

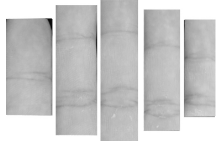

(i)

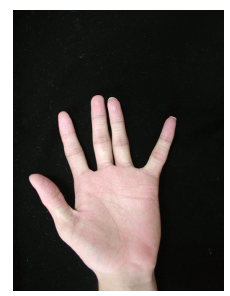

(b)

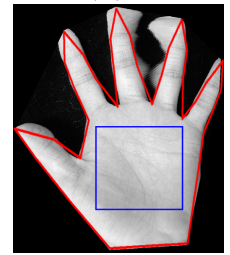

(f)

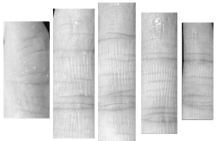

(j)

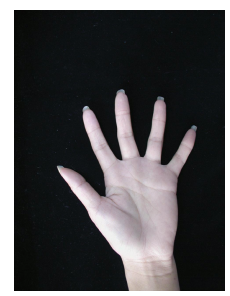

(c)

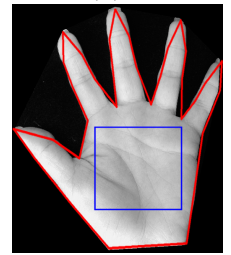

(g)

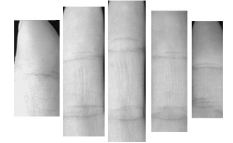

(k)

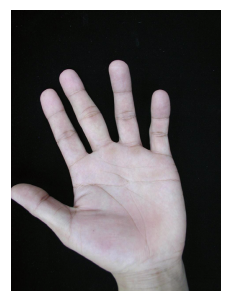

(d)

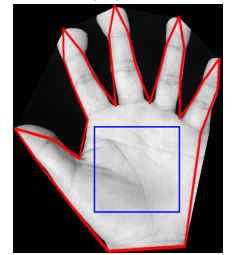

(h)

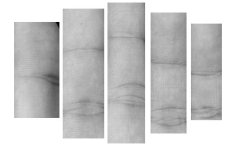

(1)

Fig. 3. Image warping result: the first row shows four sample original hand images; the second row denotes corresponding warping results, where the lines denote the mean shape obtained by averaging all samples and squares indicate the palm regions; and the last row demonstrates the segmented fingers of each hand

In low resolution hand skin images, there exist numerous randomly distributed negative lines. In our earlier work, we have demonstrated that Orthogonal Line Ordinal Features (OLOF) is capable of establishing stable and powerful representation for palmprint [6]. Due to the similarity of skin of palm and that of fingers, it is reasonable to adopt the same feature here for both of the two methods. The similarity of features is measured by Hamming distance. 


\section{Experimental Results}

Experiments were conducted on a popular database, namely the UST hand image database 11. It contains 5, 660 hand images captured from 287 subjects, i. e. 10 images per hand. In the experiments, each hand is considered to be one class, thus the total number of classes is 574. UST Database distinguishes itself from PolyU Palmprint Database and CASIA Palmprint Database (which is captured in our laboratory) in that the whole hand is imaged, which makes it more suitable for our proposed method. However, in some of the images, such as those shown in Fig. 4, it is very difficult to extract precise hand contour or locate landmarks, we manually remove these samples, finally producing a database of 5, 497 samples.

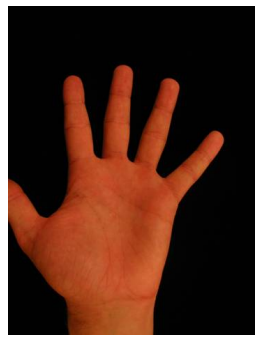

(a)

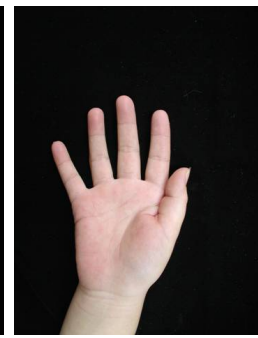

(b)

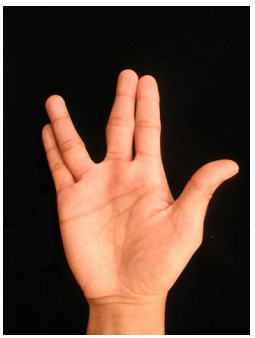

(c)

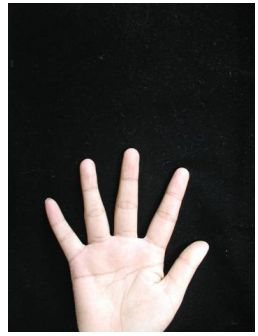

(d)

Fig. 4. Examples of bad Samples, in which reasonable hand contour is difficult to extract

During matching process, each pair of samples from the same person is matched to form the intra-class matching. Since a small portion of samples is removed, the number of intra-class matching is reduced to 25,830. To reduce the number of inter-class matching, 3 samples from each class are randomly chosen, producing $574 \times 573 \times 3 \times 3 \times 0.5=1,480,059$ inter-class matching. For fusion based method, a manually decided weight is selected for each part, and weighted sum of matching scores from different parts are adopted to make the final decision. The resulting recognition performance is illustrated in Fig. 5.

From the experimental results, we observed that fusion based method performs much better than holistic approach. This is reasonable considering that holistic method is actually a straight-forward feature level fusion scheme in that features from palm region and fingers are spatially arranged to form a higher dimension feature. However, the flexibility of fine tuning preprocessing error during matching stage, which is implemented in fusion based method by matching several horizontally or vertically switched features, is sacrificed.

The matching performance of different fingers indicates that index and middle are most reliable for matching, while thumb and little are worst among five fingers. The underlying reason for them is a little different. The feature vector for little finger is much smaller, therefore the overall discriminant power is low. However, thumb suffers most from hand pose change, in consequence, its recognition performance is sensitive to pose change and landmark localization noise. 


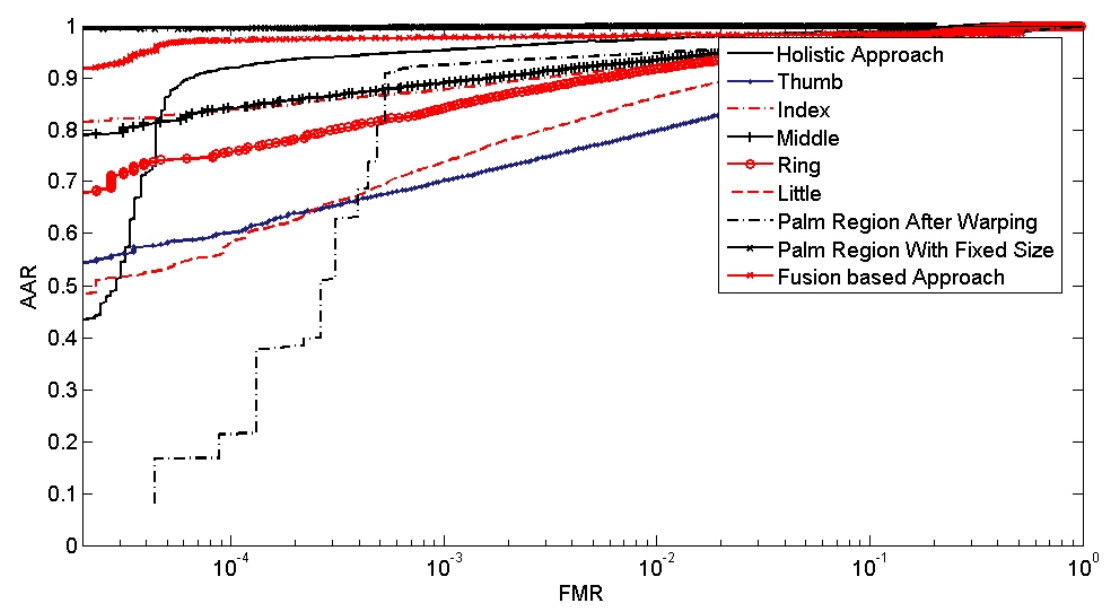

Fig. 5. Receiver Operation Characteristic Curve of proposed methods

In Fig. 5] the curve labeled with palmprint with fixed size corresponds to the matching result by locating a predefined size palm region from the hand image. The performance of fusion based method is a little lower than this nonwarping version approach. Although there is no improvement regarding recognition rate, our proposed methods do better in practical case where the scale of hands changes. Notice that the palm region based method by its own does not perform well, there are several reasons for this observation:

- While normalizing scale change, the warping process decreases the distinctiveness of hand itself. Notice that during image warping process, principal lines, and knuckles tend to be mapped to the similar region in the target images. What's more, right hand images are flipped horizontally. The consequence is that the discriminating power of warped images tends to degrade comparing with those in the literature, where a fixed size palm regions are usually selected. However, in our application scenario, a fixed size palmprint region of interest is obviously impossible because the distance between hand and camera varies.

- Non-perfect landmark localization. The precision of landmark localization is essential to warping. Visual inspection of warped images indicates that a mis-localization of 10 pixels will cause a large region of texture to stretch, which introduces negative effect to recognition performance.

\section{Conclusion and Discussion}

Our ultimate purpose is to develop a system which is capable of recognizing an individual once he (or she) naturally stretch his (or her) hand in front of camera. To achieve this goal, a pose-, scale-, translation-, and rotation- invariant image preprocessing and matching scheme is critical. The work presented in this paper serves as the first step towards this goal. 
The contribution of this paper is three folds. Firstly, the concept of handprint is proposed, which explores larger hand inner surface; Secondly, two preprocessing schemes to deal with hand pose variation are proposed to provide better user experience; Finally, textural information of finger skin is proved to be distinctive.

Although the performance is not as good as traditional palmprint-based method at current stage, there is still a large space for improvement. To benefit more from the proposed scheme, efforts should be made in the following issues:

- Automatic landmark localization. Human involvement is still necessary in wrist localization. During manual localization of wrist, global hand shape, underlying bone structure and texture cues all contribute to human judgement, which implies that wrist can not be automatically located only by contour information. In the light of this observation, automatic wrist localization may be realized with a two-step strategy: first coarsely locate the wrist using shape and geometry cues such as curvature, then refine the results with textual analysis approach.

- Better hand texture warping method. When the thumb moves from and to the palm, the region from thumb root to wrist changed dramatically. Since this movement is caused by complex mechanism which involves in many bones and muscles, it is almost impossible to fully compensate for the deformation. Similar phenomena can be observed when the fingers moves relative to each other but the deformation is less significant. Better warping method which better reflects the nature of hand skin variation may help to improve the recognition performance. Although the possibility of improvement exists, we still have to keep in mind that capturing local variations composed of occlusions, foreshortening and shadowing caused by fine scale geometry change of human skin can not be tackled only by image warping, which essentially provides no more information than the original image 10 .

Acknowledgments. This work is funded by research grants from the National Basic Research Pro- gram (Grant No. 2004CB318110), the Natural Science Foundation of China (Grant No. 60335010, 60121302, 60275003, 60332010, 6982510560605008) and the Chinese Academy of Sciences.

\section{References}

1. Yörük, E., Dutağaci, H., Sankur, B.: Hand Biometrics. Image and Vision Comptuting 24, 483-497 (2006)

2. Kumar, A., Shen, H.C.: Palmprint Identification using PalmCodes. In: Intl. Conf. Image and Graphics. IEEE, Los Alamitos (2004)

3. Kong, A.W.-K., Zhang, D.: Competitive Coding Scheme for Palmprint Verification. In: Intl. Conf. on Pattern Recognition, vol. 1, pp. 520-523 (2004)

4. Xiong, W., Toh, K.-A., Yau, W.-Y., Jiang, X.: Model-guided Deformable Hand Shape Recognition Without Positioning Aids. Patern Recognition 38, 1651-1664 (2005)

5. Kong, A.W.-K., Zhang, D.: Feature Level Fusion for Effective Palmprint Authentication. In: Intl. Conf. Biometric Authentication (2004) 
6. Sun, Z., Tan, T., Wang, Y., Li, S.Z.: Ordinal Palmprint Recognition for Personal Identification. Proc. of Computer Vision and Patrern Reccognitin (2005)

7. Ribaric, S., Fratric, I.: A Biometrics Identification System Based on Eigenpalm and Eigenfinger Features. IEEE Trans. on Pattern Analysis and Machine Intelligence 27(11), 1698-1709 (2005)

8. Wu, J., Qiu, Z.: A Hierarchical Palmprint Identification Method Using Hand Geometry and Grayscale Distribution Features. In: Intl. Conf. on Pattern Recognition, vol. 4, pp. 409-412 (2005)

9. Wolberg, G.: Digital Image Warping. IEEE Computer Society Press, Los Alamitos (1990)

10. Cula, G.O.G., Dana, K.J.: Skin Texture Modeling. Intl. Journal of Computer Vision 12(1) (2005)

11. UST Hand Image Database: http://visgraph.cs.ust.hk/biometrics/Visgraph_web/index.html

12. PolyU Palmprint Database: http://www. comp.polyu.edu.hk/ biometrics/ 